\title{
Circuit Implementation and Antisynchronization of an Improved Lorenz Chaotic System
}

\author{
Li Xiong, ${ }^{1,2}$ Yan-Jun Lu, ${ }^{1}$ Qi-Meng Zhang, ${ }^{1}$ and Zhi-Yu Zhang ${ }^{3}$ \\ ${ }^{1}$ School of Mechanical and Precision Instrumental Engineering, Xian University of Technology, Xi'an 710048, China \\ ${ }^{2}$ School of Physics and Electromechanical Engineering, Hexi University, Zhangye 734000, China \\ ${ }^{3}$ School of Automation and Information Engineering, Xi'an University of Technology, Xi'an 710048, China
}

Correspondence should be addressed to Yan-Jun Lu; yanjunlu@xaut.edu.cn

Received 16 July 2015; Revised 16 November 2015; Accepted 22 November 2015

Academic Editor: Gyuhae Park

Copyright (C) 2016 Li Xiong et al. This is an open access article distributed under the Creative Commons Attribution License, which permits unrestricted use, distribution, and reproduction in any medium, provided the original work is properly cited.

\begin{abstract}
An improved Lorenz chaotic system is proposed, making it into a circuit which is easy to be implemented by using some basic electronic components. The antisynchronization error systems can be asymptotically stabilized at the origin with three different methods which are proposed to control the improved Lorenz system. Theoretical analyses and simulation results are given to demonstrate the feasibility and effectiveness of these proposed schemes. Then the hardware circuit for the proposed Lorenz system is implemented by repeated optimization design. Experimental results show that the circuit has good comprehensive performance.
\end{abstract}

\section{Introduction}

Since 1990, the American scholars Pecora and Carroll proposed a drive-response synchronization scheme [1] and later the chaotic synchronization was implemented in the electronic circuit [2], which greatly inspired all the chaos researchers and accelerated the pace of chaos research. In recent years, due to the chaotic movement containing huge amount of information, the chaos theory has penetrated into the whole human sciences. It has a very attractive application prospect in many fields such as mathematics, physics, biological medicine, mechanical engineering, economics, and signal processing. Many researchers have turned their attention to the study of chaos which aroused great interest and enthusiasm of scholars. It makes the study of chaotic control and synchronization become the significant progress field in chaos research. So far, the researchers have done a wide and deep research on chaotic synchronization and control. They proposed and implemented the generalized synchronization, coupled synchronization, adaptive synchronization, synchronization based on state observer, and other different types of chaotic synchronization [3-11]. Work [12] implemented the robust control for a class of modified Duffing equations, and several control methods were developed for the set-point control and the trajectory tracking of a modified Duffing equation. Work [13] proposed an adaptive sliding-modeobserver-based fault reconstruction scheme for nonlinear systems with parametric uncertainties, which can be implemented online. In [14], Lyapunov based adaptive control scheme was proposed for a class of MIMO nonlinear systems by state and output feedback, and asymptotic convergence of the tracking error was established in the presence of uncertainty. With its further study, some scholars expand the concept of synchronization. They proposed the concept of chaotic antisynchronization and realized the antisynchronization [15-19] of different chaotic systems with different methods. Works $[15,16]$ implemented the antisynchronization of two identical chaotic systems with coupled and nondirectional coupling, respectively. Work [17] realized the antisynchronization of a class of chaotic systems based on the state observer. In work [18], synchronization and antisynchronization were implemented for a new hyperchaotic Lü system with uncertain parameters via the passive control technique. Therefore, the study on the antisynchronization of chaotic systems is of great potential market value.

In fact, it is not realistic to design most chaotic systems into chaotic signal generator. The reasons are mainly as 


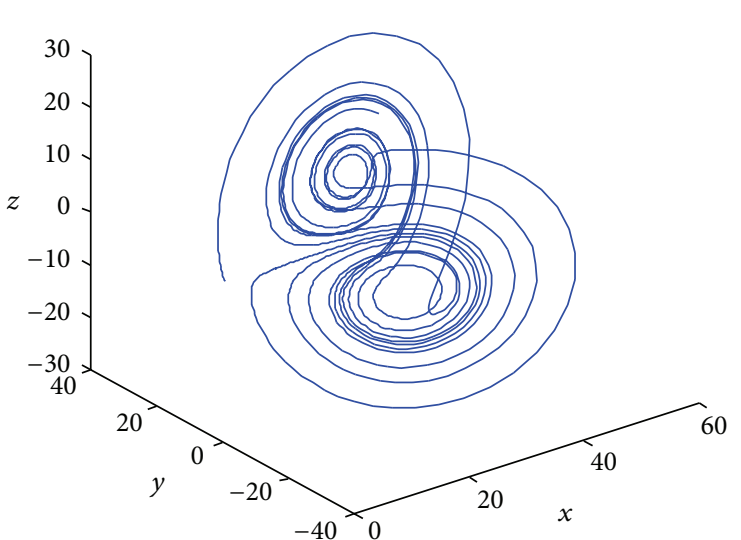

(a)

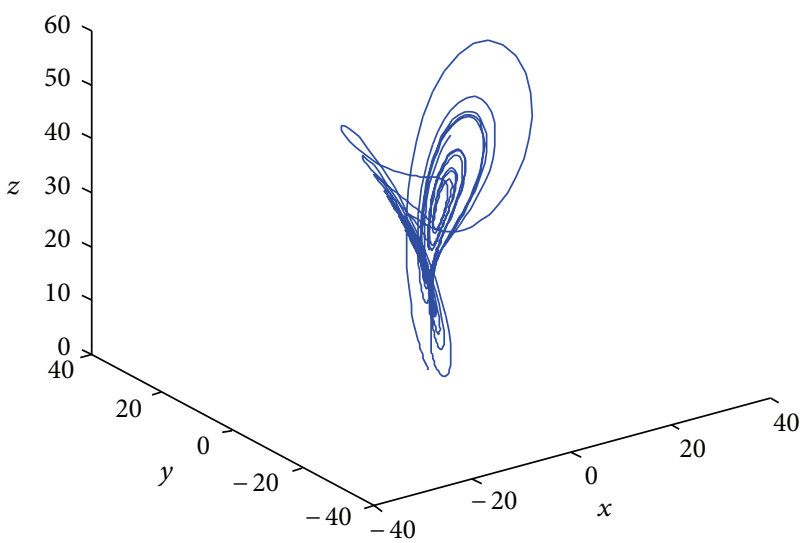

(b)

FIGURE 1: Strange attractors of the original Lorenz chaotic system.

follows: the chaotic system is complex and difficult to operate (such as neural network [6]); the nonlinear devices are too many to grasp the accuracy (such as Lorenz system [20]); the stability is not high (such as Chua's circuit [21, 22]); another reason is the difficulty of synchronization, small frequency band range, and high production cost. Therefore, the design of the chaotic circuit [20-27] is still a great challenge, and there are many problems worth studying. In practice, numerical solutions of some systems are not able to be implemented by using some circuit components. Thus, the ranges of these variables need to be adjusted appropriately in order to fully satisfy the requirements of circuit design in practical application.

The main contributions of this paper include the following: firstly, in this paper, the original Lorenz equation is improved, which is easy to be implemented by using basic electronic components. Secondly, three different methods such as active control, adaptive control, and control based on state observer are adopted to control the proposed Lorenz chaotic system. With active control method, the antisynchronization error system can be asymptotically stabilized at the origin. With adaptive control method, the antisynchronization error system can be adjusted adaptively to stabilize the proposed Lorenz system at the origin by selecting the appropriate control function. With control based on state observer method, the antisynchronization error system can be asymptotically stabilized at the origin without calculating conditional Lyapunov exponent. Thirdly, in the following work, the optimal chaotic circuit of the improved Lorenz system is proposed and implemented by using some basic electronic components containing analog multipliers, operational amplifiers, resistors, and capacitors. However, due to the fact that implementation of Lorenz analog electronic circuit needs analog multipliers, it needs higher accuracy requirements on the parameters of the electronic components. In addition, we compare the advantages and disadvantages of these proposed antisynchronization schemes in practical applications.

\section{An Improved Lorenz System}

The original Lorenz equation is described as follows:

$$
\begin{aligned}
& \dot{x}=\sigma(y-x), \\
& \dot{y}=\rho x-y-x z, \\
& \dot{z}=x y-\beta z .
\end{aligned}
$$

When choosing $\sigma=10, \rho=28$, and $\beta=8 / 3$, system (1) is chaotic. Its three-dimensional phase diagrams of strange attractors are shown in Figure 1.

By analyzing the simulation results, it can be concluded that the display range of $x$-axis is from -25 to $25, y$-axis shows the range from -25 to +25 , and $z$-axis shows the range from 0 to +50 . The product term of $x y$ is nearly 400 and $x z$ is nearly 700 . In practical electronic circuits, the working voltage range of electronic components is generally from $-15 \mathrm{~V}$ to $+15 \mathrm{~V}$, and good linear working range is from $-10 \mathrm{~V}$ to $+10 \mathrm{~V}$. Therefore, the numerical solutions of the original Lorenz equation cannot be implemented by using basic electronic components. That is to say, if the above data values are understood as voltage volts, the design will inevitably fail. Thus, in practical application, it is often necessary to adjust these variables appropriately. The introduction of new variables is described as follows:

$$
\begin{aligned}
u & =\frac{x}{10}, \\
v & =\frac{y}{10}, \\
w & =\frac{z}{30} .
\end{aligned}
$$

Lorenz equation (1) to be represented by $u, v$, and $w$ becomes

$$
\begin{aligned}
\dot{u} & =\sigma(v-u), \\
\dot{v} & =\rho u-v-30 u w, \\
\dot{w} & =3.3 u v-\beta w .
\end{aligned}
$$




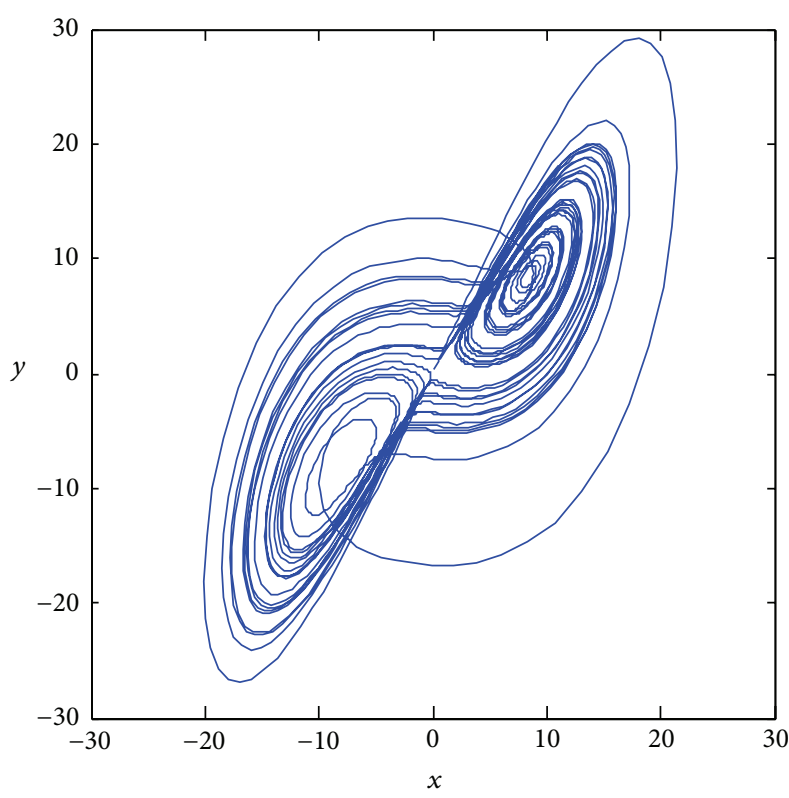

(a) $x-y$ phase diagram

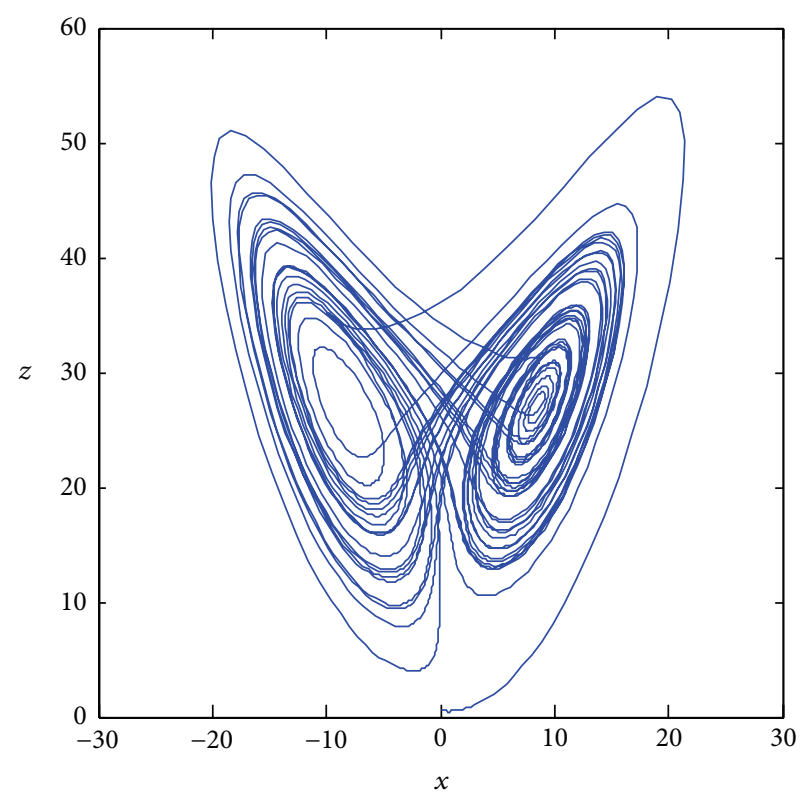

(b) $x-z$ phase diagram

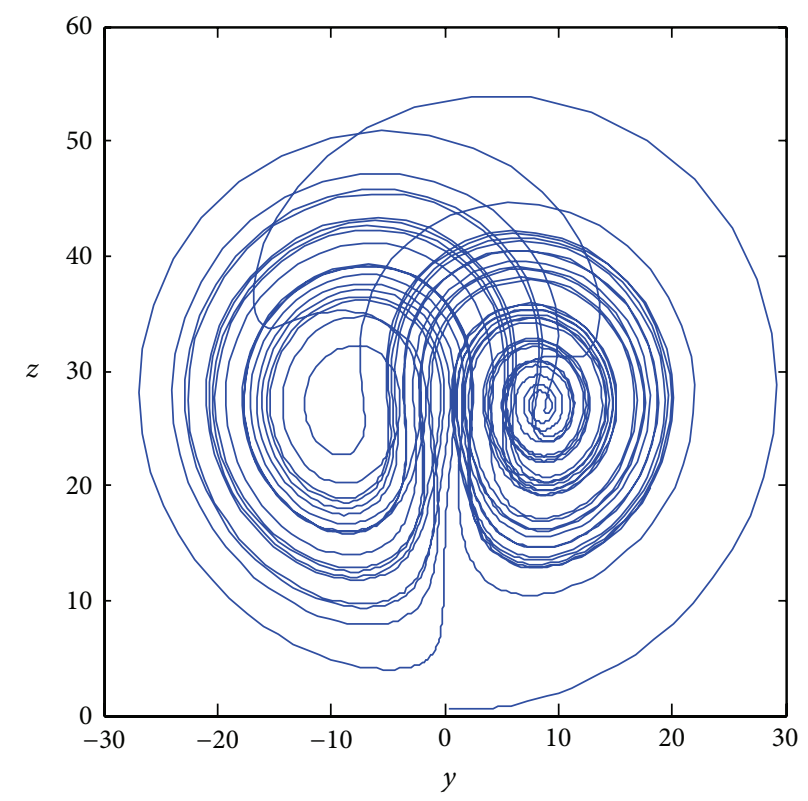

(c) $y-z$ phase diagram

FIgURE 2: Phase diagrams of the improved Lorenz chaotic system.

That is,

$$
\begin{aligned}
& \dot{x}=\sigma(y-x), \\
& \dot{y}=\rho x-y-30 x z, \\
& \dot{z}=3.3 x y-\beta z .
\end{aligned}
$$

Substituting the specific parameter values, (4) is described as follows:

$$
\begin{aligned}
& \dot{x}=-10 x+10 y, \\
& \dot{y}=28 x-y-30 x z, \\
& \dot{z}=3.3 x y-\left(\frac{8}{3}\right) z .
\end{aligned}
$$

This is the improved Lorenz chaotic system. Simulation phase diagrams are shown in Figure 2. It can be noticed 
from Figure 2 that the numerical range of each parameter is within $-10 \mathrm{~V}$ to $+10 \mathrm{~V}$. In other words, the improved Lorenz system fully conforms to the requirements of circuit design in practical application, which has a certain economic value.

\section{Antisynchronization of the Improved Lorenz Chaotic System with Three Methods}

3.1. Active Control Method. The drive system is described as follows:

$$
\begin{aligned}
& \dot{x}_{1}=a\left(x_{2}-x_{1}\right), \\
& \dot{x}_{2}=b x_{1}-x_{2}-30 x_{1} x_{3}, \\
& \dot{x}_{3}=3.3 x_{1} x_{2}-c x_{3} .
\end{aligned}
$$

When choosing $a=10, b=28$, and $c=8 / 3$, system (6) is chaotic. The response system is described as follows:

$$
\begin{aligned}
& \dot{y}_{1}=a\left(y_{2}-y_{1}\right)+u_{1}, \\
& \dot{y}_{2}=b y_{1}-y_{2}-30 y_{1} y_{3}+u_{2}, \\
& \dot{y}_{3}=3.3 y_{1} y_{2}-c y_{3}+u_{3},
\end{aligned}
$$

where $u_{1}, u_{2}$, and $u_{3}$ are the controllers [12].

The antisynchronization error is defined as $\dot{e}=\dot{x}+\dot{y}$; then the antisynchronization error of system (6) and system (7) is described as follows:

$$
\begin{aligned}
& \dot{e}_{1}=a\left(e_{2}-e_{1}\right)+u_{1}, \\
& \dot{e}_{2}=b e_{1}-e_{2}-30 x_{1} x_{3}-30 y_{1} y_{3}+u_{2}, \\
& \dot{e}_{3}=3.3 x_{1} x_{2}+3.3 y_{1} y_{2}-c e_{3}+u_{3} .
\end{aligned}
$$

The controller is constructed as follows:

$$
\begin{aligned}
& u_{1}=-a\left(e_{2}-e_{1}\right)-k_{1} e_{1}, \\
& u_{2}=30 x_{1} x_{3}+30 y_{1} y_{3}+e_{2}-b e_{1}-k_{2} e_{2}, \\
& u_{3}=c e_{3}-3.3 x_{1} x_{2}-3.3 y_{1} y_{2}-k_{3} e_{3},
\end{aligned}
$$

where $k_{i}>0(i=1,2,3)$, for controlling the speed of antisynchronization. Substituting (9) into (8), we can obtain

$$
\begin{aligned}
& \dot{e}_{1}=-k_{1} e_{1}, \\
& \dot{e}_{2}=-k_{2} e_{2}, \\
& \dot{e}_{3}=-k_{3} e_{3} .
\end{aligned}
$$

It can be concluded from (10) that the eigenvalues of the Jacobi matrix of the error system are negative. It can be seen from the Lyapunov stability theory that the antisynchronization error system is asymptotically stable at the origin. That is, $\lim _{t \rightarrow \infty}|e(t)| \rightarrow 0$. Therefore, it proves that the antisynchronization between the drive system and the response system is achieved.

Computer simulations are made by using Matlab and Simulink. The initial values of the drive system are chosen as $x_{1}(0)=2, x_{2}(0)=-3$, and $x_{3}(0)=6$. The initial values of the response system are chosen as $y_{1}(0)=-3$, $y_{2}(0)=5$, and $y_{3}(0)=-4$. The control gains are chosen as $k_{1}=k_{2}=k_{3}=10$. The antisynchronization error curves are shown in Figures 3(a), 3(b), and 3(c). As can be seen from these figures, for less than 1 second, the antisynchronization errors $e_{1}, e_{2}$, and $e_{3}$ can be asymptotically stabilized at the origin. Figure $3(\mathrm{~d})$ shows the timing diagram of $x_{1}-y_{1}$. It can be seen that the waveforms of the two systems are just opposite, and the antisynchronization is implemented. It is proved that the method is simple and practical, and the antisynchronization time is very short. As is well known in the practical application of engineering, the less control signal is, the more easily the hardware circuit of the control process is implemented. Therefore, the proposed scheme is easier to be implemented by hardware circuit because of its low control signal and low cost. However, the anti-interference ability of this method is weaker.

3.2. Adaptive Control Method. Adaptive antisynchronization involves using adaptive control technology to automatically adjust some parameters of the system to implement the antisynchronization of two systems. The application of this method has two conditions: one is that at least one or more parameters of the system can be obtained; another is for the desired orbit; these parameters are known. The adjustment of the parameters of the system also depends on two factors: the difference between the output of the system and the corresponding variable of the desired orbit; the error between the controlled parameter values and the response parameter values. Adaptive control method is suitable for the chaotic system with parameter mismatch, which has a very strong anti-interference ability and universality. Therefore, it has a certain practical value for the study of adaptive antisynchronization.

We choose system (6) as the drive system, and system (7) is chosen as the response system. Similarly, the antisynchronization error is defined as $\dot{e}=\dot{x}+\dot{y}$; then system (8) is the antisynchronization error system. By selecting the appropriate control function [12] $u_{i}(i=1,2,3)$, the antisynchronization error system is adaptively adjusted to achieve antisynchronization with multiple parameters.

The controller is constructed as follows:

$$
\begin{aligned}
& u_{1}=-a_{1}\left(e_{2}-e_{1}\right)-k_{1} e_{1}, \\
& u_{2}=30 x_{1} x_{3}+30 y_{1} y_{3}+e_{2}-b_{1} e_{1}-k_{2} e_{2}, \\
& u_{3}=c_{1} e_{3}-3.3 x_{1} x_{2}-3.3 y_{1} y_{2}-k_{3} e_{3} .
\end{aligned}
$$

The parameter adaptive law is constructed as follows:

$$
\begin{aligned}
& \dot{a}_{1}=\left(e_{2}-e_{1}\right) e_{1}, \\
& \dot{b}_{1}=e_{1} e_{2}, \\
& \dot{c}_{1}=-e_{3}^{2} .
\end{aligned}
$$




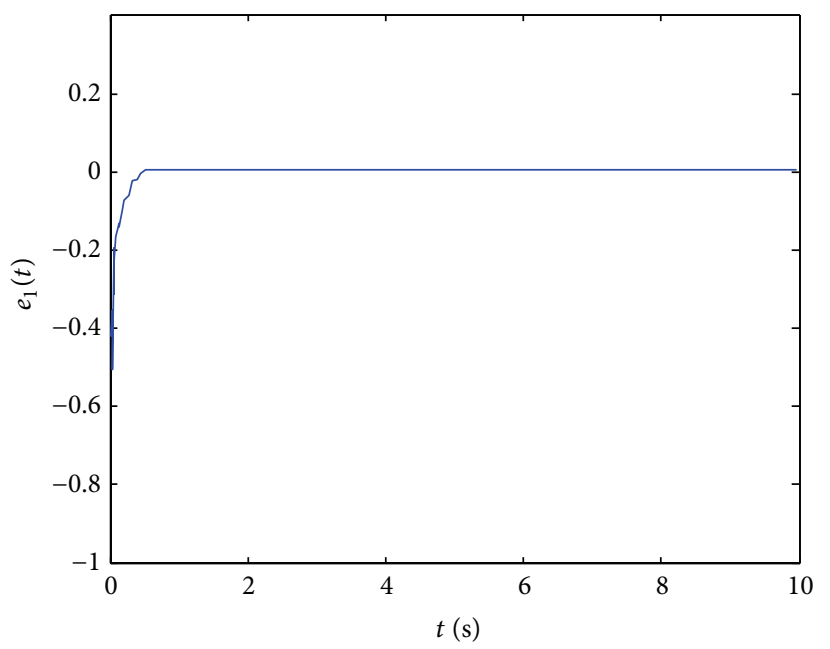

(a) Antisynchronization error $e_{1}$

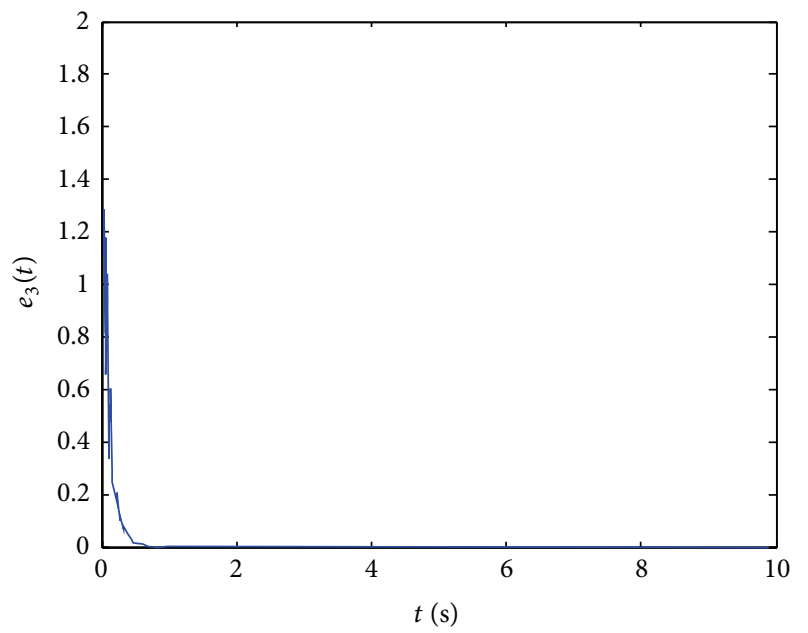

(c) Antisynchronization error $e_{3}$

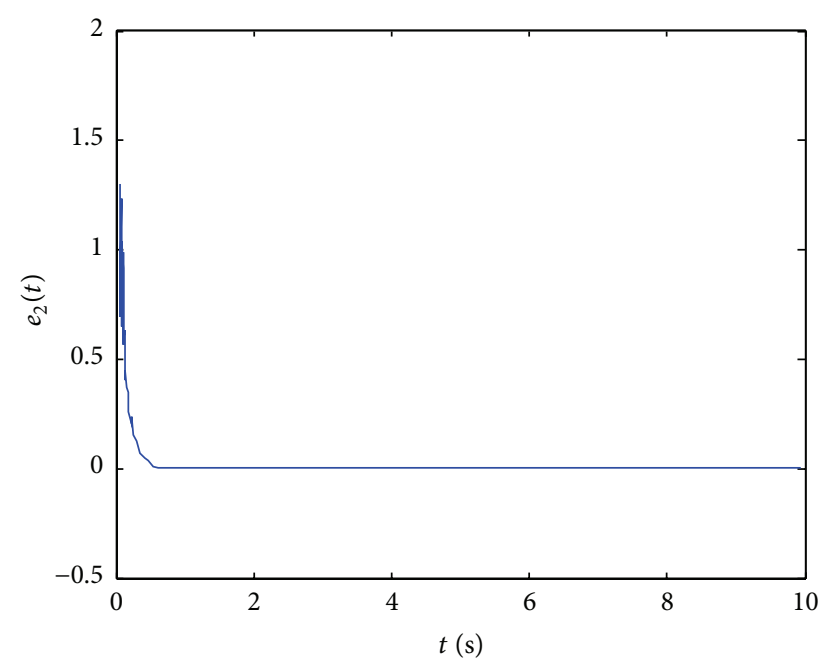

(b) Antisynchronization error $e_{2}$
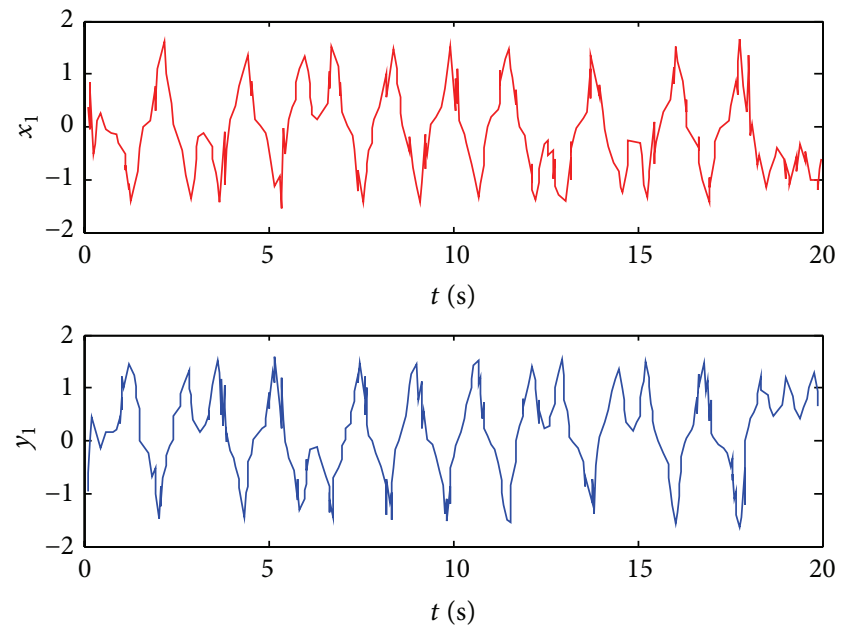

(d) Antisynchronized waveforms of $x_{1}-y_{1}$

FIGURE 3: Antisynchronization error curves and waveforms of active control.

Substituting (11) into (8), we can obtain

$$
\begin{aligned}
& \dot{e}_{1}=\left(a-a_{1}\right)\left(e_{2}-e_{1}\right)-k_{1} e_{1}, \\
& \dot{e}_{2}=\left(b-b_{1}\right) e_{1}-k_{2} e_{2}, \\
& \dot{e}_{3}=\left(c_{1}-c\right) e_{3}-k_{3} e_{3},
\end{aligned}
$$

where $a_{1}, b_{1}$, and $c_{1}$ are the control parameters to be determined. Order parameter errors $X=a-a_{1}, Y=b-b_{1}$, and $Z=c-c_{1}$; then the antisynchronization error system (13) becomes

$$
\begin{aligned}
& \dot{e}_{1}=A\left(e_{2}-e_{1}\right)-k_{1} e_{1}, \\
& \dot{e}_{2}=B e_{1}-k_{2} e_{2}, \\
& \dot{e}_{3}=-C e_{3}-k_{3} e_{3} .
\end{aligned}
$$

To facilitate the control design, the Lyapunov function $V[13$, 14] is defined as follows:

$$
V=\frac{\left(e_{1}^{2}+e_{2}^{2}+e_{3}^{2}+X^{2}+Y^{2}+Z^{2}\right)}{2} .
$$

Obviously, $V$ is a positive definite function. It follows from (15) that

$$
\begin{aligned}
\dot{V}= & e_{1} \dot{e}_{1}+e_{2} \dot{e}_{2}+e_{3} \dot{e}_{3}+X \dot{X}+Y \dot{Y}+Z \dot{Z} \\
= & e_{1}\left(X e_{2}-X e_{1}-k_{1} e_{1}\right)+e_{2}\left(Y e_{1}-k_{2} e_{2}\right) \\
& +e_{3}\left(-c e_{3}-k_{3} e_{3}\right)+X \dot{X}+Y \dot{Y}+Z \dot{Z} \\
= & -k_{1} e_{1}^{2}-k_{2} e_{2}^{2}-k_{3} e_{3}^{2}+X e_{1}\left(e_{2}-e_{1}\right)+Y e_{1} e_{2} \\
& -Z e_{3}^{2}+X \dot{X}+Y \dot{Y}+Z \dot{Z} .
\end{aligned}
$$




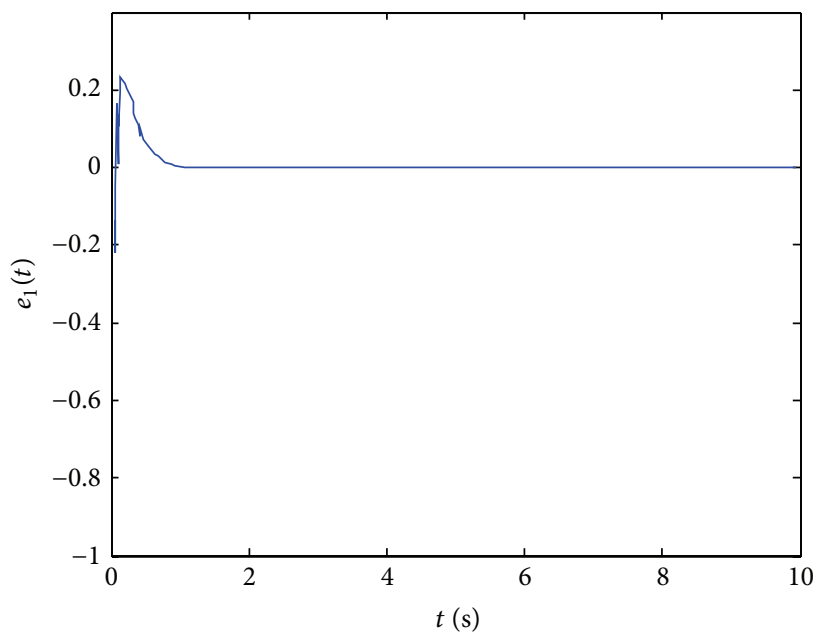

(a) Antisynchronization error $e_{1}$

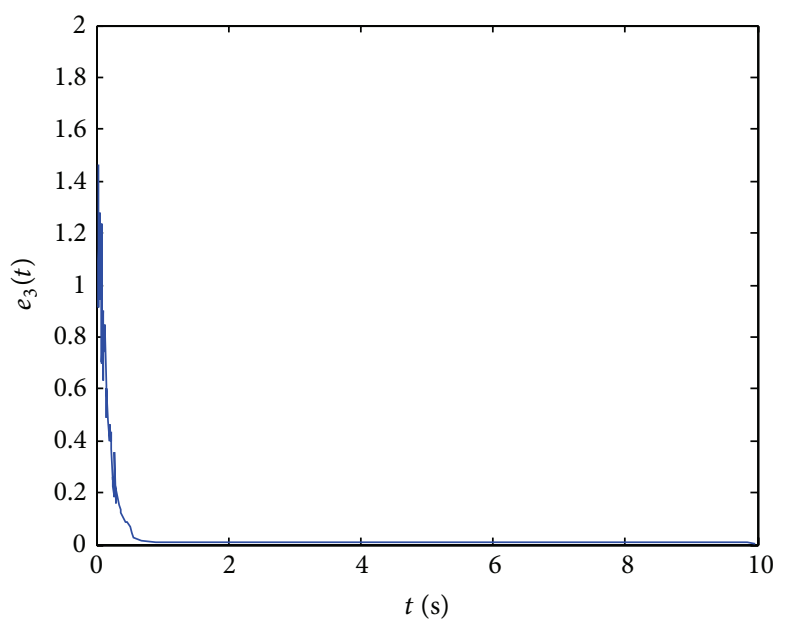

(c) Antisynchronization error $e_{3}$

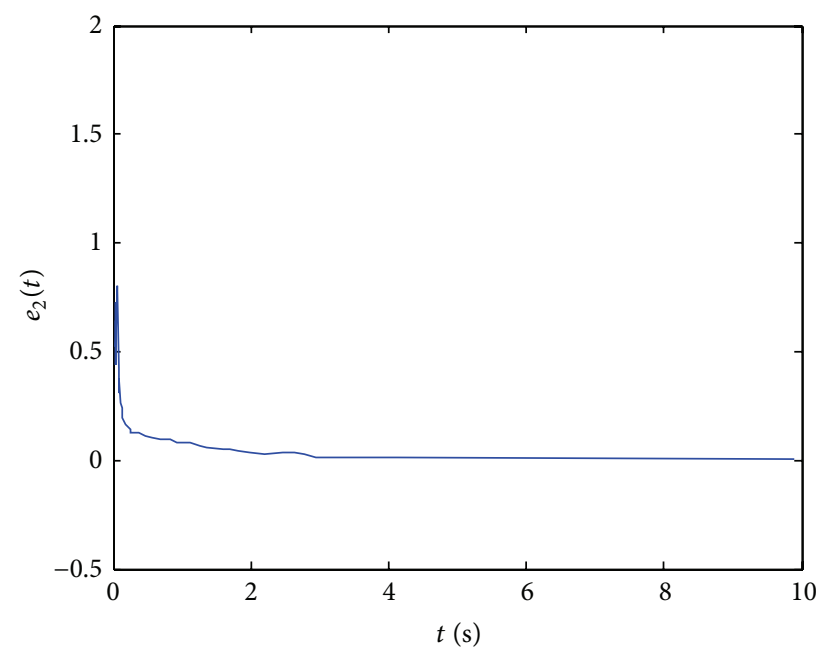

(b) Antisynchronization error $e_{2}$
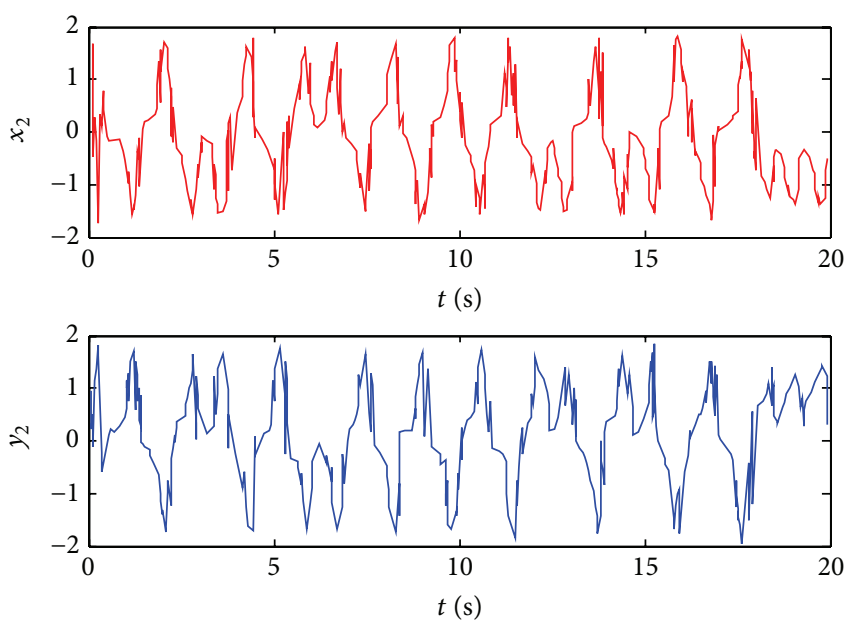

(d) Antisynchronized waveforms of $x_{2}-y_{2}$

FIGURE 4: Adaptive antisynchronization error curves and waveforms.

Let

$$
\begin{aligned}
& \dot{X}=e_{1}^{2}-e_{1} e_{2}, \\
& \dot{Y}=-e_{1} e_{2}, \\
& \dot{Z}=e_{3}^{2} .
\end{aligned}
$$

Then, we can obtain $\dot{V}=-k_{1} e_{1}^{2}-k_{2} e_{2}^{2}-k_{3} e_{3}^{2} \leq 0$; thus $\dot{V}$ is negative semidefinite. According to Lyapunov stability theory, if $V$ is positive definite and $\dot{V}$ is negative semidefinite, then the system is consistent and stable at the origin of the equilibrium state in the sense of Lyapunov. Therefore, the antisynchronization error system is asymptotically stable at the origin; that is, $\lim _{t \rightarrow \infty}|e(t)| \rightarrow 0$. It proves that the adaptive antisynchronization between the drive system and the response system is achieved.

In the following simulation, the initial values of the drive system are chosen as $x_{1}(0)=2, x_{2}(0)=-3$, and $x_{3}(0)=6$. The initial values of the response system are chosen as $y_{1}(0)=$ $-3, y_{2}(0)=5, y_{3}(0)=-4, a_{1}=5, b_{1}=6$, and $c_{1}=7$.
The control gains are chosen as $k_{1}=k_{2}=k_{3}=10$. The adaptive antisynchronization error curves are shown in Figures 4(a), 4(b), and 4(c). As can be seen from the figures, in a very short period of time, the antisynchronization errors $e_{1}, e_{2}$, and $e_{3}$ can be adjusted adaptively to asymptotically stabilize at the origin by selecting the appropriate control function. Figure 4(d) shows the timing diagram. It is proved that the above method is effective for the implementation of adaptive antisynchronization. However, the amount of control and control costs are increased because of the adaptive adjustment of multiple parameters, which makes the hardware implementation complicated.

3.3. Control Based on State Observer. The so-called state observer can be achieved physically with the observation system of the same order of dynamic systems. It is driven by the output signal of the observed system (which is physically measured) and realizes that all state variables or outputs are close to the state variables or outputs of the observed system. And the state observer is designed to provide 
the estimates for full system states only using fewer output signals which are available for measurement. It is not necessary to calculate conditional Lyapunov exponent for the antisynchronization method based on state observer, and it is easy to be implemented in engineering. The mathematical definition and design scheme of the antisynchronization state observer are given below.

Consider the following nonlinear system:

$$
\dot{x}=A x+B_{i} f_{i}(x)+C, \quad i=1,2,3, \ldots,
$$

where $A \in R^{n \times n}, B_{i} \in R^{n \times n}(i=1,2,3, \ldots)$, and $f: R^{n} \rightarrow$ $R^{m}(m \leq n)$, and $f_{i}(x)$ is nonlinear mapping, $A x$ is a linear part for the system, $B_{i} f_{i}(x)$ is a nonlinear part for the system, and $B_{i}$ is a constant matrix for the system. The output of (18) is described as follows:

$$
u_{1 i}=K x+f_{i}(x), \quad i=1,2,3, \ldots,
$$

where $K$ is undetermined coefficient. The nonlinear system is composed of (18) and (19):

$$
\begin{aligned}
\dot{x} & =A x+B_{i} f_{i}(x)+C, \\
u_{1 i} & =K x+f_{i}(x), \\
& i=1,2,3, \ldots .
\end{aligned}
$$

The state observer of (20) can be constructed according to the state observer design method of the control theory:

$$
\begin{aligned}
\dot{y} & =A y+B_{i} f_{i}(y)-C-B_{i}\left(u_{1 i}+u_{2 i}\right), \\
u_{2 i} & =K y+f_{i}(y),
\end{aligned}
$$

$$
i=1,2,3, \ldots
$$

The antisynchronization error is defined as $\dot{e}=\dot{x}+\dot{y}$. Then the antisynchronization error system is described as follows:

$$
\begin{aligned}
\dot{e}= & A(x+y)+B_{i}\left[f_{i}(x)+f_{i}(y)\right]-B_{i}\left(u_{1 i}+u_{2 i}\right) \\
= & A(x+y)+B_{i}\left[f_{i}(x)+f_{i}(y)\right] \\
& -B_{i}\left[K x+f_{i}(x)+K y+f_{i}(y)\right] \\
= & A(x+y)-B_{i} K x-B_{i} K y=A e-B_{i} K e \\
= & {\left[A-\left(B_{1}+B_{2}+B_{3} \cdots\right) K\right] e . }
\end{aligned}
$$

If all the eigenvalues of the real matrix $\left[A-\left(B_{1}+B_{2}+\right.\right.$ $\left.\left.B_{3} \cdots\right) K\right]$ are negative, the antisynchronization error system is asymptotically stable. That is, $\lim _{t \rightarrow \infty}|e(t)| \rightarrow 0$, and the antisynchronization is implemented. Through configuring the pole position of the matrix $\left[A-\left(B_{1}+B_{2}+B_{3} \cdots\right) K\right]$, all the eigenvalues of the matrix can be satisfied to have a negative real component. Suppose $\left[A-\left(B_{1}+B_{2}+B_{3} \cdots\right) K\right]$ has a characteristic value of $\lambda$; matrix $K$ is determined by builtin function $K=\operatorname{place}(A, B, \lambda)$ in Matlab. In engineering, the dynamic performance of the system is often estimated by the dominant pole. As a result, a pair of dominant poles and a pair of complex poles away from the dominant real poles should be selected for the simulation.
Choosing system (6) as the drive system, and in (18),

$$
\begin{aligned}
A & =\left[\begin{array}{ccc}
-a & a & 0 \\
b & -1 & 0 \\
0 & 0 & -c
\end{array}\right], \\
B_{1} & =\left[\begin{array}{c}
0 \\
30 \\
0
\end{array}\right], \\
B_{2} & =\left[\begin{array}{c}
0 \\
0 \\
3.3
\end{array}\right],
\end{aligned}
$$

where $f_{1}(x)=-x_{1} x_{3}, f_{2}(x)=x_{1} x_{2}, u_{11}=K x+f_{1}(x)$, and $u_{12}=K x+f_{2}(x)$. And in (19), $f_{1}(y)=-y_{1} y_{3}, f_{2}(y)=y_{1} y_{2}$, $u_{21}=K y+f_{1}(y)$, and $u_{22}=K y+f_{2}(y)$.

Then the response system is described as follows:

$$
\begin{aligned}
& \dot{y}_{1}=a\left(y_{2}-y_{1}\right), \\
& \dot{y}_{2}=b y_{1}-y_{2}-30\left(u_{11}+u_{21}\right), \\
& \dot{y}_{3}=-c y_{3}-3.3\left(u_{12}+u_{22}\right),
\end{aligned}
$$

so system (24) is the antisynchronous state observer of system (6).

Some computer simulations are provided to verify the feasibility of the proposed antisynchronization scheme. In the simulation, let $\lambda=(-5,-20+i,-20-i)$; then $K=(1.4692,1.1247,-0.7294)$. The initial values of the drive system are chosen as $x_{1}(0)=5, x_{2}(0)=3$, and $x_{3}(0)=$ -1 . The initial values of the response system are chosen as $y_{1}(0)=-3, y_{2}(0)=-6$, and $y_{3}(0)=2$. The antisynchronization error curves are shown in Figures 5(a), 5(b), and 5(c). As can be seen from the figures, in a short period of time, the antisynchronization errors $e_{1}, e_{2}$, and $e_{3}$ can be asymptotically stabilized at the origin without calculating conditional Lyapunov exponent. Figure 5(d) shows the timing diagram. It proves that the proposed method is simple and the antisynchronization performance is good.

\section{Circuit Implementation for the Improved Lorenz System}

In order to sufficiently testify the dynamic behavior of the proposed Lorenz system, an analog circuit is designed by using resistors, capacitors, analog operational amplifiers, and analog multipliers. The original circuit design of Lorenz system is shown in Figure 6 according to (5). Although it can implement the function of (5), it is not the most optimal circuit. In order to obtain the most optimal circuit, the circuit shown in Figure 6 needs to be optimized. The basic idea of optimization design is to simplify, minimize, and merge the circuit by utilizing the basic knowledge of circuit theory under the condition of without changing the circuit function. The optimization procedures are designed as follows. 


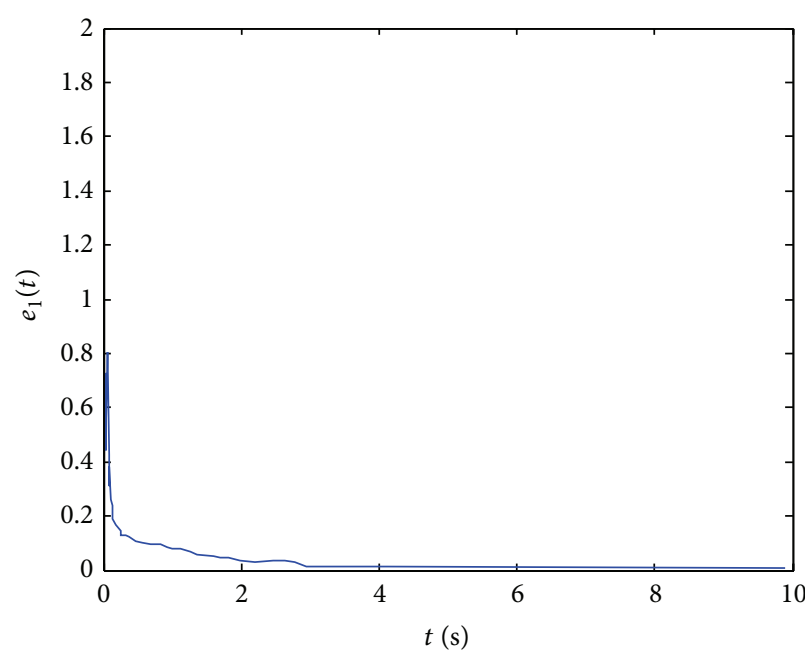

(a) Antisynchronization error $e_{1}$

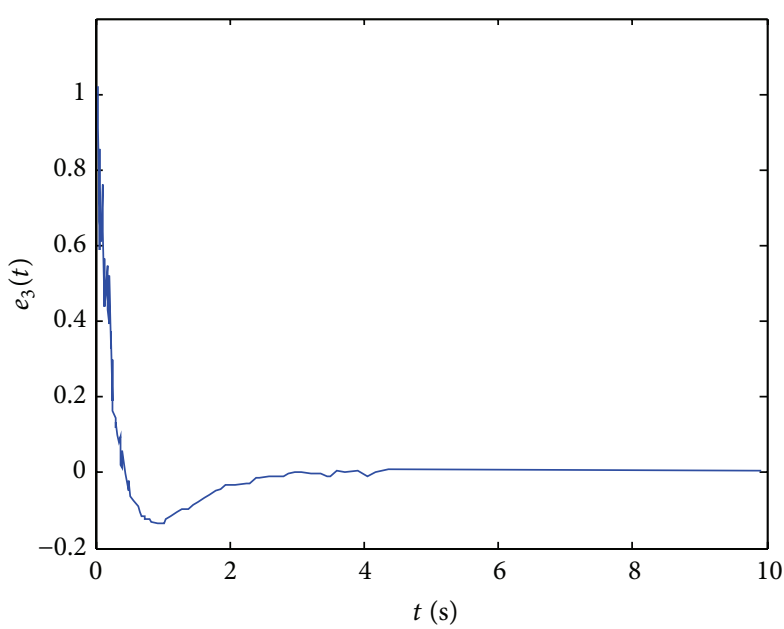

(c) Antisynchronization error $e_{3}$

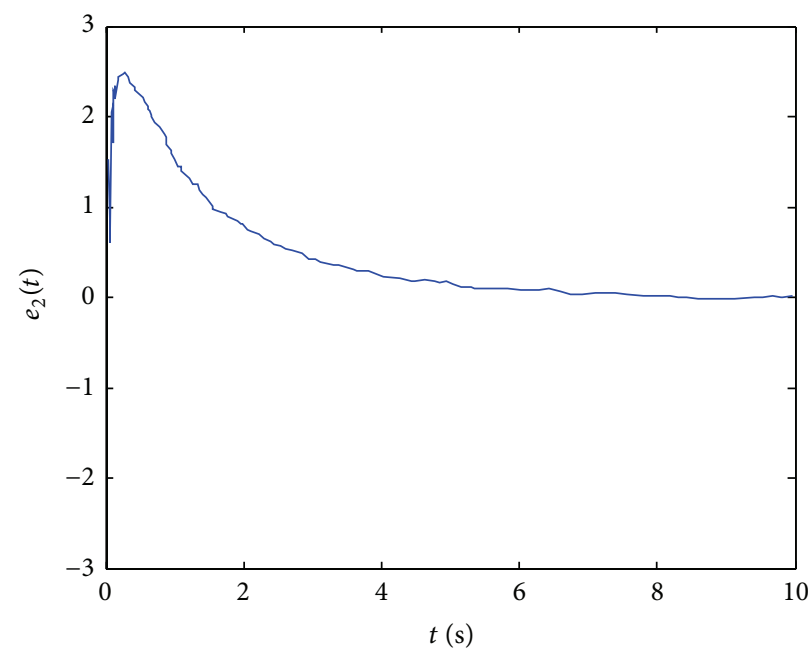

(b) Antisynchronization error $e_{2}$
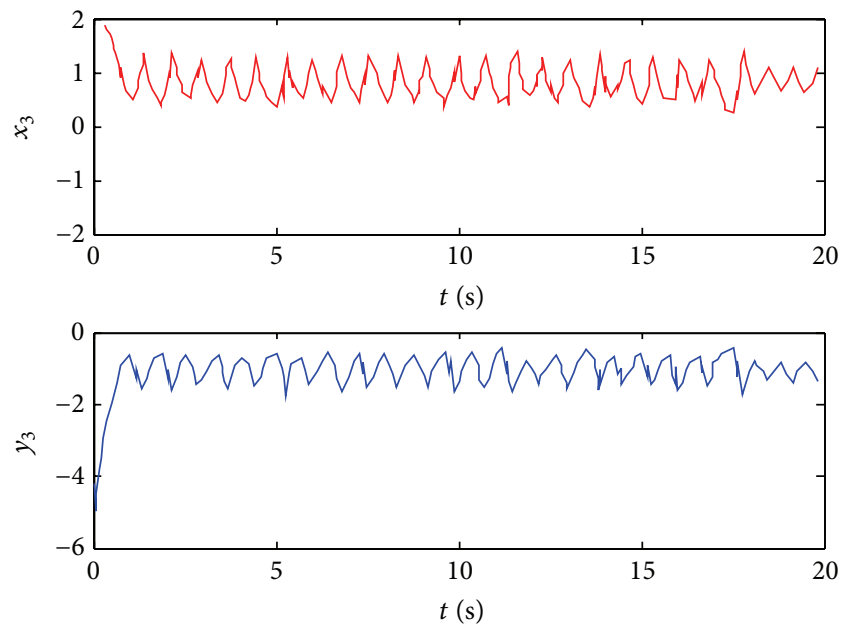

(d) Antisynchronized waveforms of $x_{3}-y_{3}$

FIGURE 5: Antisynchronization error curves and waveforms based on the state observer.

(1) The function of $A_{1}$ is an input $x$ and an output $-x$, which is enlarged -1 times. $A_{2}$ is enlarged -1 times through $R_{3}$ and then back into $x$. It is equal to doing nothing. Therefore $R_{1}, R_{2}, A_{1}$, and $R_{3}$ should be replaced by a resistor connected to the reverse input terminal of $A_{3}$. To satisfy the one magnified 10 times for $A_{3}$, the alternative resistance $R=10 \mathrm{k} \Omega$.

(2) The input of $R_{10}$ is $y$, which is $-y$ enlarged -1 times through $A_{5}$. Then it is again enlarged -1 times through $R_{13}$ and $A_{6}$ and back into $y$. It is equal to doing nothing. Therefore, $R_{10}$ can be directly connected to the reverse input terminal of $A_{7}$. To satisfy the one magnified 1 time, the alternative resistance $R=100 \mathrm{k} \Omega$.

(3) The inputs of MUL1 are $x$ and $z$, and output is $0.1 x z$, which is $30 y$ magnified 300 times through $A_{8}$. So $0.1 x z$ can be directly connected to $A_{7}$ through an alternative resistance. To satisfy the differential equation of $100 \mathrm{k} \Omega / R=30 x z / 0.1 x z$, the alternative resistance $R=330 \Omega$.

(4) Now the input resistance of $A_{5}$ is only $R_{9}$. The input of $A_{4}$ is $x$ and output is $-x$, which is enlarged -28 times. Then it is enlarged -1 times through $R_{9}$ and $A_{5}$ and enlarged -1 times through $R_{13}$ and $A_{6}$, which becomes $28 x$.

(5) The inputs of MUL2 are $x$ and $y$, and output is 0.1, which is $3.3 x y$ enlarged 33 times through $A_{10}$. So $0.1 x y$ can be directly connected to $R_{23}$.

(6) The function of $A_{9}$ is an input $z$. It is enlarged $-8 / 3$ times and then again enlarged -1 times through $R_{22}$ and $A_{11}$, which becomes $(8 / 3) z$. Therefore $R_{18}, R_{19}$, $A_{9}$, and $R_{22}$ can be replaced by a resistor. To satisfy the one magnified $8 / 3$ times, the alternative resistance $R=37.5 \mathrm{k} \Omega$. 


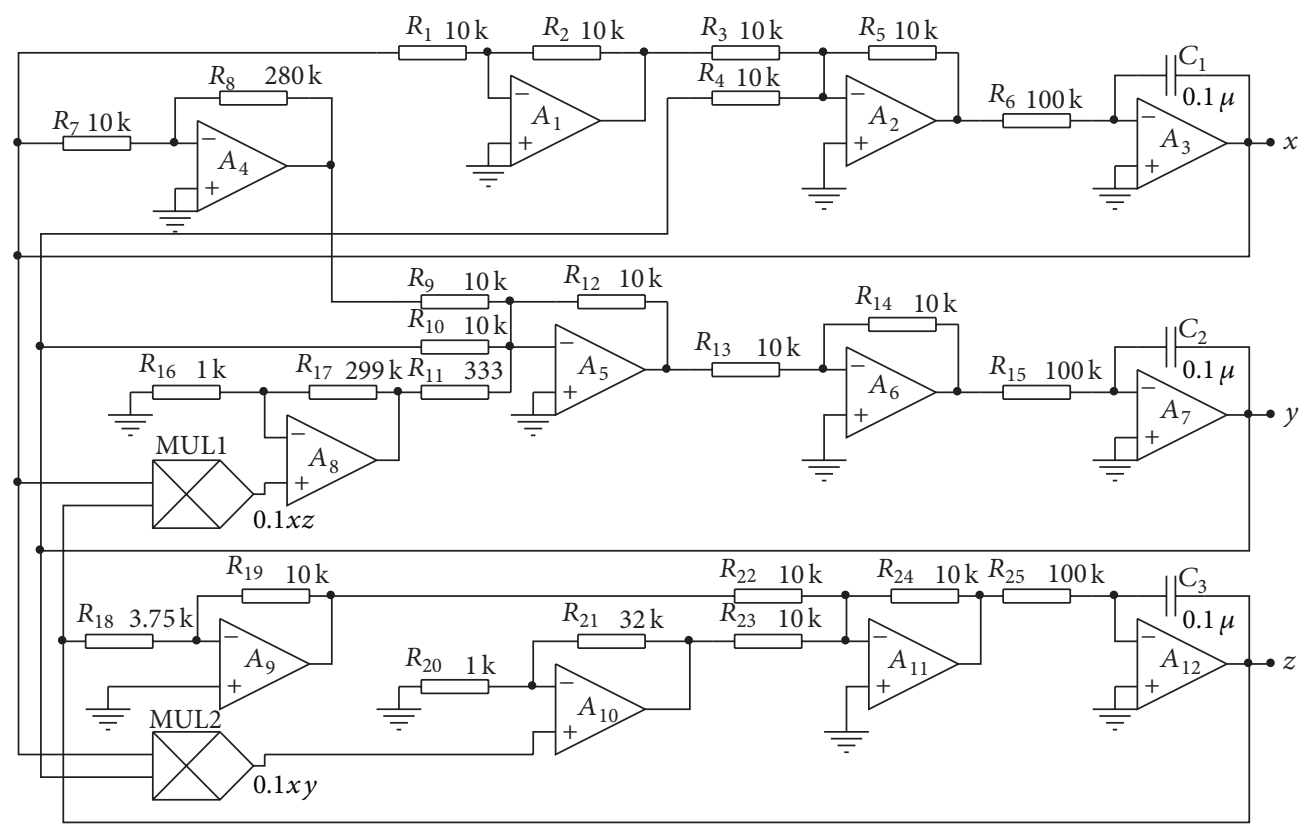

FIGURE 6: The original circuit design.

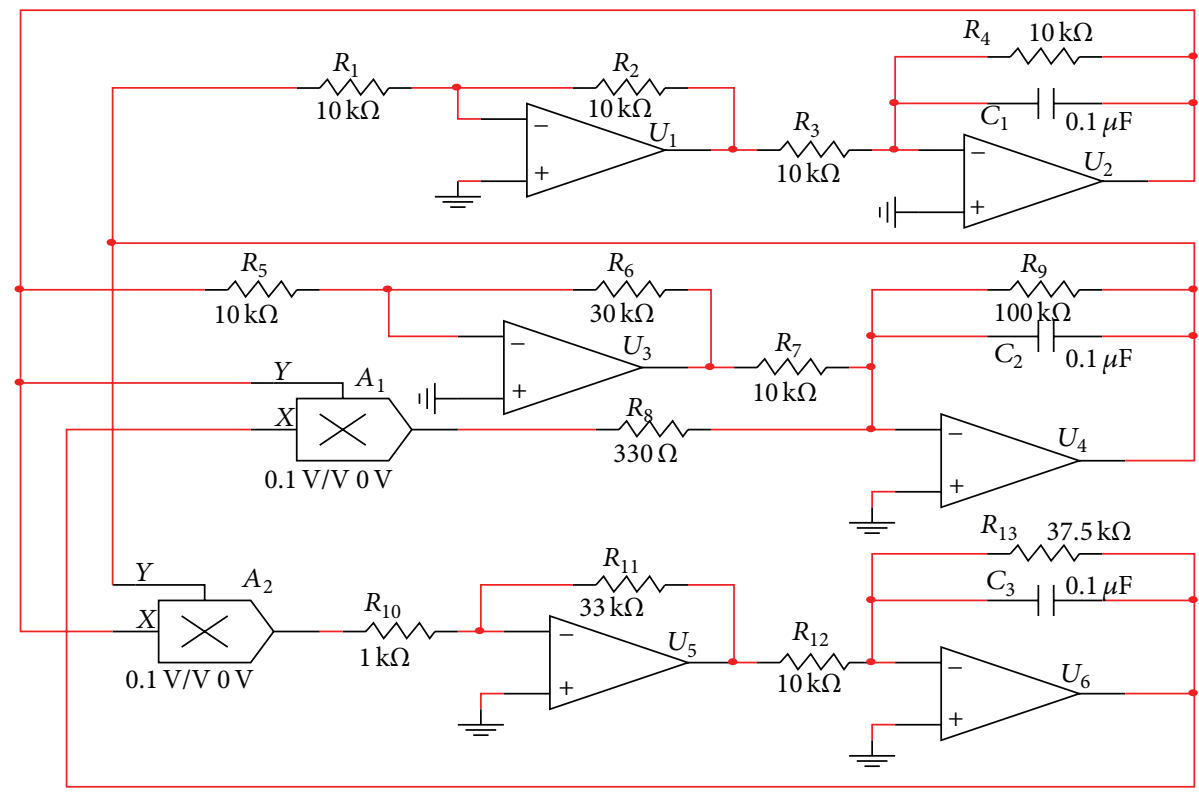

FIGURE 7: The final optimization circuit.

The final optimization design result of Lorenz chaotic circuit is shown in Figure 7 by Multisim. As can be seen from Figure 7, the number of operational amplifiers is reduced from 12 to 6 , and other passive components are also correspondingly reduced. Through optimization, the complexity of the circuit, the thermal noise of the total resistance, and the total error of the circuit can be reduced. Meanwhile, the cost can be reduced. After optimizing the circuit is simple, easy to debug, and suitable for mass production. Figure 8 shows the simulation phase diagram.
For the circuit of Figure 7, we make the actual circuit and the experimental results are shown in Figures 9 and 10. The output waveform pictures are shown in Figure 9. The output phase diagram pictures are shown in Figure 10.

\section{Conclusion}

In this paper, an improved Lorenz chaotic system has been proposed. This is because the numerical solutions of original Lorenz system are not easy to be implemented by using 


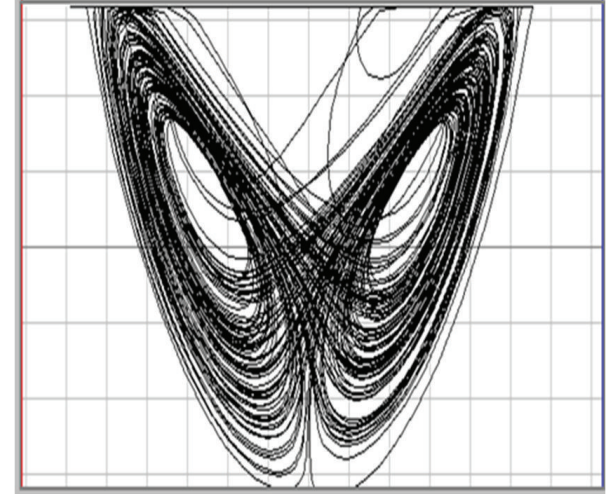

FIGURE 8: Simulation phase diagram.

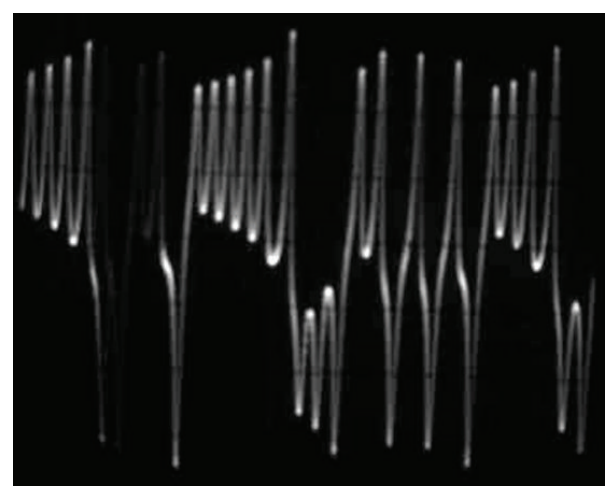

(a) $x$ waveform diagram

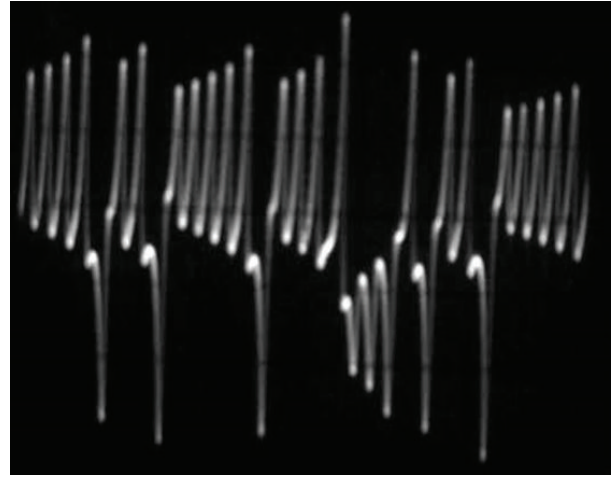

(b) $y$ waveform diagram

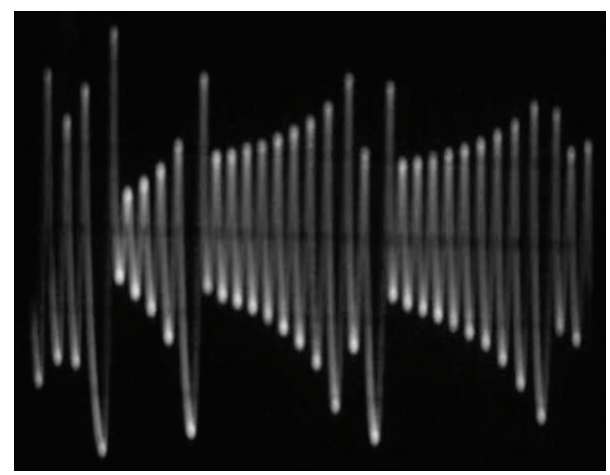

(c) $z$ waveform diagram

Figure 9: The output waveform pictures of improved Lorenz circuit experiment board.

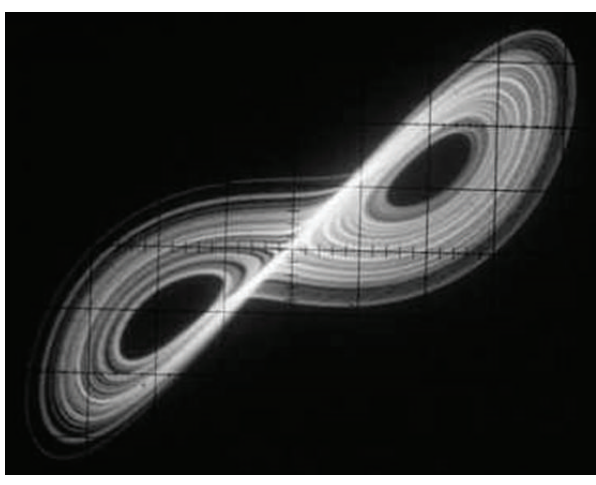

(a) $x-y$ phase diagram

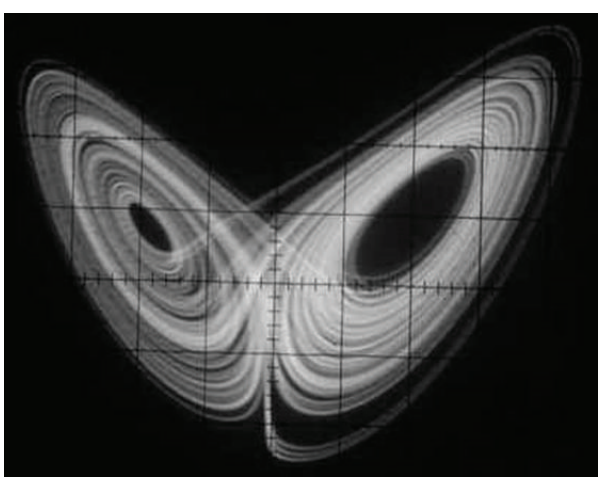

(b) $x-z$ phase diagram

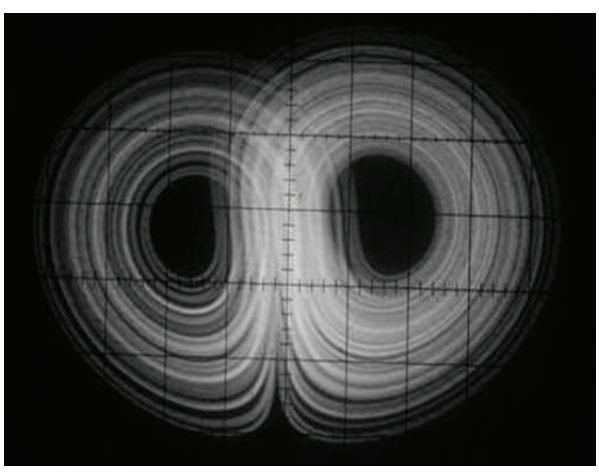

(c) $z-y$ phase diagram

FIGURE 10: The output phase diagram pictures of improved Lorenz circuit experiment board.

basic electronic components. The values of passive components have certain range, and the operating voltages of active electronic devices also have certain scope in actual electronic circuit. Then several control methods have been developed to implement the antisynchronization of improved Lorenz system. The active control method is easier to be implemented by hardware circuit because of its low control signal and low cost. The advantage of the adaptive control method is that it does not need to know the specific dynamic equations of the system, so that the antisynchronization of the system can be obtained again from the mismatch of the parameters of the system by adaptive adjustment. Therefore, it has very strong anti-interference ability and universality. At the same time, because of the large number of the selected 
controllers, the difficulty and complexity of hardware circuit design are increased greatly, so it is not conducive to the practical application of the project. The control method based on state observer has the property of keeping the response system in chaos state and does not need to calculate the Lyapunov exponent. It is rather suitable to be applied in most of chaotic decryption mechanism. By the use of the state observer in chaotic secure communication, the number of signals transmitted through the public channel can be greatly decreased in order to further guarantee the security and good robustness. But it has a relatively long time to implement antisynchronization. Theory analyses and simulation results prove the feasibility and practicability of these proposed antisynchronization schemes.

Furthermore, some basic electronic components are used to implement the proposed Lorenz chaotic circuit. Chaotic synchronization or antisynchronization is the premise and foundation for secure communication [10, 22]; therefore, the research of this paper has some guidance for chaotic secure communication circuit. In our future work, the proposed control methods and circuit design scheme may be further made in chaotic secure communication.

\section{Conflict of Interests}

The authors declare that there is no conflict of interests regarding the publication of this paper.

\section{Acknowledgments}

This work was financially supported by the National Natural Science Foundation of China (Grant no. 51505375), Open project of State Key Laboratory of Mechanical Transmission (Grant no. SKLMT-KFKT-201510), and Scientific Research Program of Shanxi Provincial Education Department of China (15JS068 and 15JK1549).

\section{References}

[1] L. M. Pecora and T. L. Carroll, "Synchronization in chaotic systems," Physical Review Letters, vol. 64, no. 8, pp. 821-824, 1990.

[2] K. M. Cuomo and A. V. Oppenheim, "Circuit implementation of synchronized chaos with applications to communications," Physical Review Letters, vol. 71, no. 1, pp. 65-68, 1993.

[3] M. Boutayeb, M. Darouach, and H. Rafaralahy, "Generalized state-space observers for chaotic synchronization and secure communication," IEEE Transactions on Circuits and Systems. I. Fundamental Theory and Applications, vol. 49, no. 3, pp. 345349, 2002.

[4] G. R. Chen and J. H. Lü, Dynamical Analyses, Control and Synchronization of the Lorenz System Family, Science Press, Beijing, China, 2003.

[5] X. Wu and J.-A. Lu, "Adaptive control of uncertain Lü system," Chaos, Solitons \& Fractals, vol. 22, no. 2, pp. 375-381, 2004.

[6] L.-H. Yu and J.-C. Fang, "Synchronization of chaotic neural networks based on adaptive inverse control and its applications in secure communications," Acta Physica Sinica, vol. 54, no. 9, pp. 4012-4018, 2005.
[7] M. Roopaei, B. R. Sahraei, and T.-C. Lin, "Adaptive sliding mode control in a novel class of chaotic systems," Communications in Nonlinear Science and Numerical Simulation, vol. 15, no. 12, pp. 4158-4170, 2010.

[8] X.-J. Wu and H.-T. Lü, "Adaptive generalized function projective lag synchronization of different chaotic systems with fully uncertain parameters," Chaos, Solitons \& Fractals, vol. 44, no. 10, pp. 802-810, 2011.

[9] S. Li, J. Yang, W.-H. Chen, and X. Chen, "Generalized extended state observer based control for systems with mismatched uncertainties," IEEE Transactions on Industrial Electronics, vol. 59, no. 12, pp. 4792-4802, 2012.

[10] M. Sheikhan, R. Shahnazi, and S. Garoucy, "Synchronization of general chaotic systems using neural controllers with application to secure communication," Neural Computing and Applications, vol. 22, no. 2, pp. 361-373, 2013.

[11] S. Das, M. Srivastava, and A. Y. Leung, "Hybrid phase synchronization between identical and nonidentical three-dimensional chaotic systems using the active control method," Nonlinear Dynamics, vol. 73, no. 4, pp. 2261-2272, 2013.

[12] X.-G. Yan, I.-M. Chen, and H.-S. Li, "Robust control for a class of modified Duffing equations," Transactions of the Institute of Measurement and Control, vol. 24, no. 4, pp. 263-275, 2002.

[13] X.-G. Yan and C. Edwards, "Adaptive sliding-mode-observerbased fault reconstruction for nonlinear systems with parametric uncertainties," IEEE Transactions on Industrial Electronics, vol. 55, no. 11, pp. 4029-4036, 2008.

[14] S. S. Ge and Z. Li, "Robust adaptive control for a class of MIMO nonlinear systems by state and output feedback," IEEE Transactions on Automatic Control, vol. 59, no. 6, pp. 1624-1629, 2014.

[15] C.-M. Kim, S. W. Rim, W.-H. Kye, J.-W. Ryu, and Y.-J. Park, "Anti-synchronization of chaotic oscillators," Physics Letters A, vol. 320, no. 1, pp. 39-46, 2003.

[16] J. Hu, S. Chen, and L. Chen, "Adaptive control for anti-synchronization of Chua's chaotic system," Physics Letters A, vol. 339, no. 6, pp. 455-460, 2005.

[17] X.-Y. Wang and X.-J. Wu, "Chaos anti-synchronization of a class of chaotic systems based on state observer design," Acta Physica Sinica, vol. 56, no. 4, pp. 1988-1993, 2007.

[18] X. Zhou, B. Kong, and H. Ding, "Synchronization and antisynchronization of a new hyperchaotic Lü system with uncertain parameters via the passive control technique," Physica Scripta, vol. 85, no. 6, Article ID 065004, 2012.

[19] C. Wang, Y. He, J. Ma, and L. Huang, "Parameters estimation, mixed synchronization, and antisynchronization in chaotic systems," Complexity, vol. 20, no. 1, pp. 64-73, 2014.

[20] J. H. Lü, G. R. Chen, and S. Celikovsky, "Bridge the gap between the Lorenz system and Chen system," International Journal of Bifurcation and Chaos, vol. 12, no. 12, pp. 2917-2928, 2002.

[21] K. Murali, S. Sinha, and W. L. Ditto, "Implementation of NOR gate by a chaotic Chua's circuit," International Journal of Bifurcation and Chaos, vol. 13, no. 9, pp. 2669-2672, 2003.

[22] W. Ni and L. Wang, "Research on chaos communication based on Chua's circuit," Computer and Modernization, vol. 64, no. 7, pp. 96-98, 2005.

[23] X. G. Zhang, Y. D. Ma, and S. L. Li, Nonlinear Circuit-Based Analysis and Design, vol. 1, Higher Education Press, Beijing, China, 2011.

[24] Z. Xu, C.-X. Liu, and T. Yang, "Study on a new chaotic system with analysis and circuit experiment," Acta Physica Sinica, vol. 59, no. 1, pp. 131-139, 2010. 
[25] T. Liangrui, L. Jing, and F. Bing, "A new chaotic system and its circuit simulation," Acta Physica Sinica, vol. 58, no. 2, pp. 785793, 2009.

[26] C. W. Wu, "Synchronization in coupled chaotic circuits and systems," World Scientific Series A, vol. 41, no. 1, pp. 157-159, 2002.

[27] B. Muthuswamy and L. O. Chua, "Simplest chaotic circuit," International Journal of Bifurcation and Chaos, vol. 20, no. 5, pp. 1567-1580, 2010. 


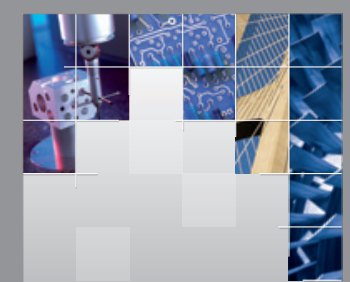

\section{Enfincering}
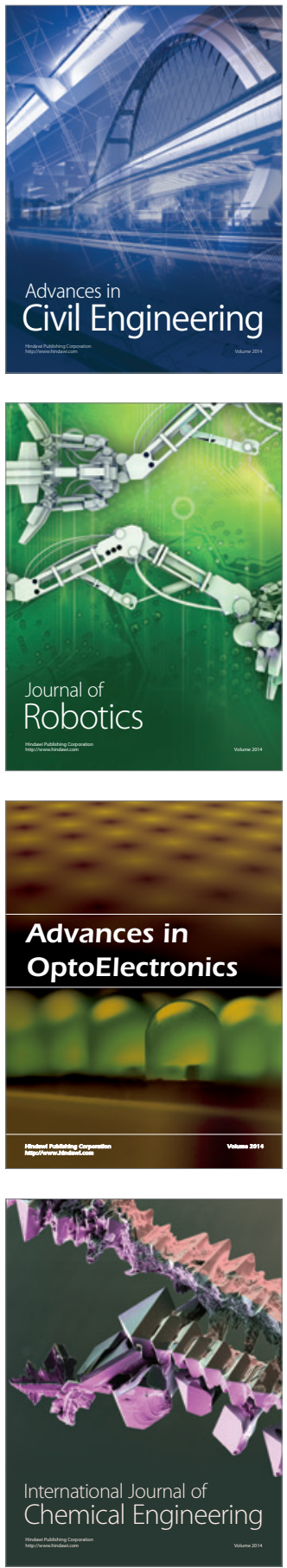

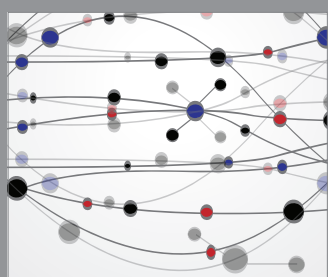

The Scientific World Journal

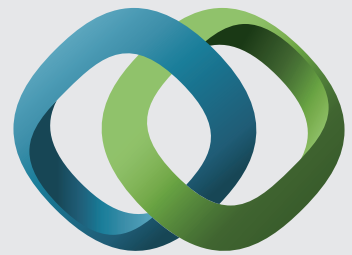

\section{Hindawi}

Submit your manuscripts at

http://www.hindawi.com
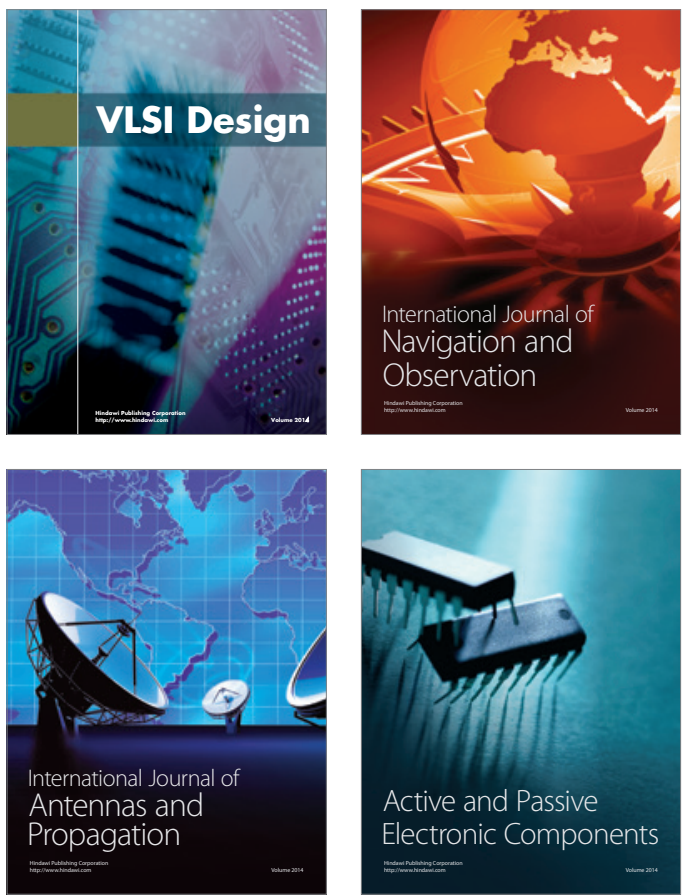
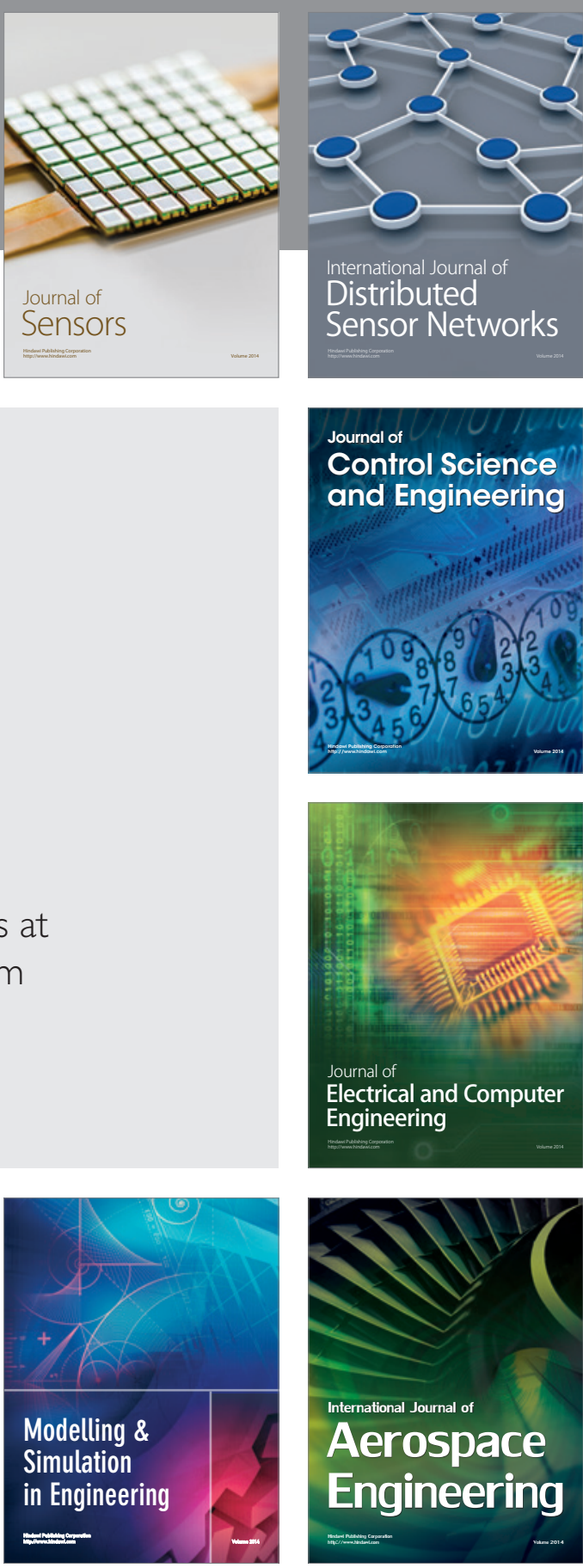

International Journal of

Distributed

Sensor Networks

Journal of

Control Science

and Engineering
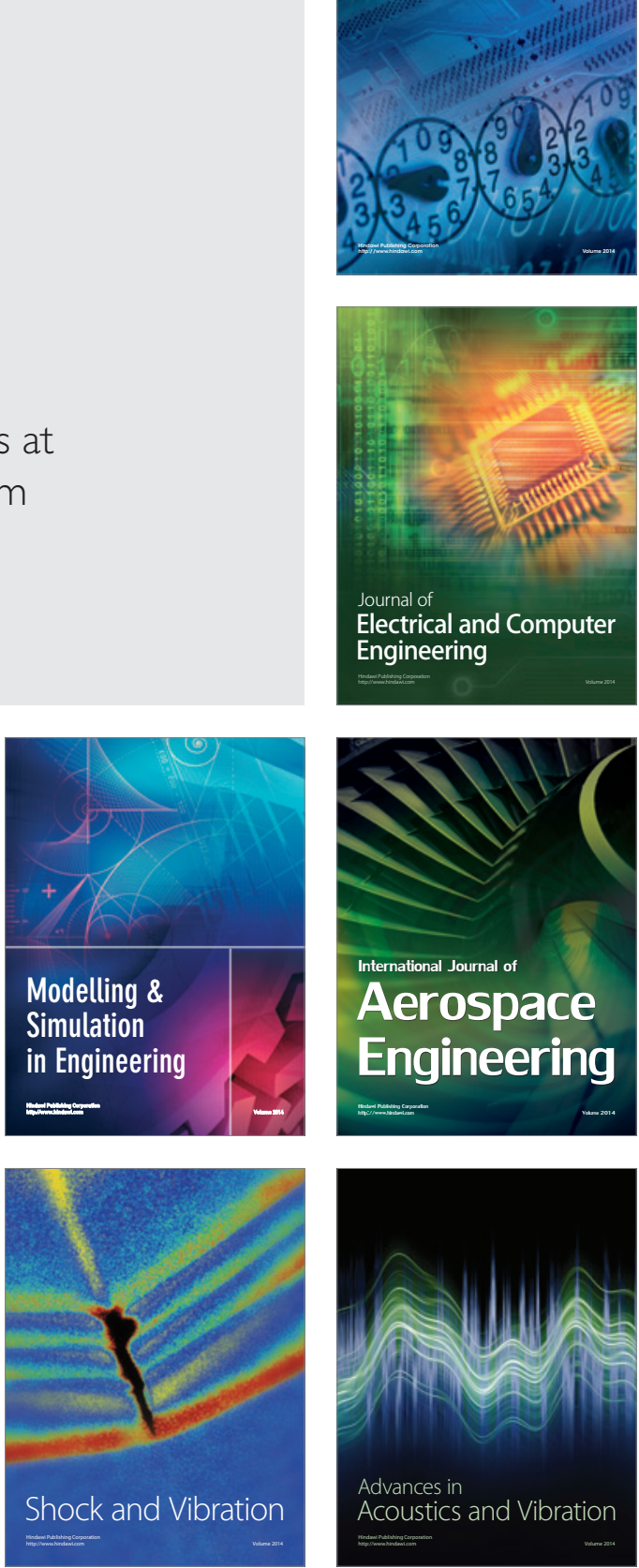